\title{
Présentation de quelques aspects de recherches récentes sur l'agitation de la mer
}

\section{Some aspects of recent research on sea waves}

\author{
PAR M. BANAL
}

ADJOIN AU DIRECTEUR DES ÉTUDES ET REGHERCHES D'ÉLECTRICITÉ DE FRANCE

\author{
Communication présentée à la Société Hydrotechnique de France le 18 juin 1959
}

L'analyse de l'agitation créée par le vent $\dot{a}$ la surface de la mer est trop complexe pour être abordée dans toute sa généralité et les études théoriques se développent dans des directions différentes et apparemment contradictoires.

On a tenté de dresser un inventaire de ces études d'après la nature des simplifications apportées par leurs auteurs aux lois complètes et exactes régissant le phénomène.

On a ainsi distingué :

- les studes relatives a la génération de la houle par le vent qui conduisent aux méthodes de prévision de la houle;

- les études tenant compte de la viscosité qui ont trait à l'action de la houle sur les fonds et les obstacles;

- la recherche des solutions périodiques des équations de l'hydrodynamique simplifiées de manière à être linéaires qui constituent encore la manière la plus générale et souvent la seule de traiter la majorité des problèmes de propagation et d'effet de la houle sur les ouvrages;

- la recherche de solutions cylindriques périodiques des équations exactes de l'hydrodynamique qui permet de traiter avec plus d'exactitude quelques problèmes particuliers;

- Ia représentation et l'étude des houles naturelles non périodiques.

On a commenté avec un peu plus de détails, d̀ propos de ce dernier genre d'étude, la représentation de la houle par un processus aléatoire.

On a montré les raisons pratiques élémentaires qui pouvaient justifier cette représentation en insistant sur le fail que l'insuffisance des données naturelles ne permettait pas de fournir des preuves formelles de validité surtout pour l'extension de cette méthode aux houles non cylindriques.
The analysis of wave disturbance generated on the surface of the sea by wind is too complex for it to be possible to tackle it in the most general fashion. Recent theoretical studies have taken different and often contradictory lines.

The author has tried to list these studies according to the simplifications made to the complete and exact laws governing the phenomenon.

The following categories are considered:

Studies concerning the generation of waves by the wind and leading to wave forecasting methods.

Studies which take viscosity effects into account and deal with the effect of wanes on the sea bed and on obstacles.

The finding of periodic solutions to linearized hydrodynamic equations. This is the most generul and often the only way of considering wawe propagation and the effects of waves on structures.

The finding of cy/indrical periodic solutions to exact hydrodynamic equations which make it possible to deal with certain special cases more accurately.

The simulation and study of natural non-periodic waves.

Fuller details are given, in connection with the last category, of the simulation of waves by random methods.

Practical elementary reasons are given which may justify simulation of this kind, and $\mathrm{em}$ phasis is laid on the fact that lack of prototype data makes it impossible to prove its validity formally, especially when the melhod is extended to non-cylindrical waves. 


\section{INTRODUCTION}

Les études relatives à l'agitation de la mer se développent dans des directions très différenles et les préoccupations de leurs auteurs peuvent même paraître contradictoires.

Nous avons essayć de dresser un inventaire de ces études en montrant comment elles se completent et à quels problèmes pratiques elles se rapportent.

Le plan que nous suivrons peut se schématiser dans le tableau ci-dessous:

\begin{tabular}{|c|c|c|}
\hline Caractéristique des études & Problèmes pratiques traités & $\begin{array}{l}\text { Développement } \\
\text { des études }\end{array}$ \\
\hline Effet du vent sur la mer. & Prévision de la houle. & Relativement récent. \\
\hline Prise en compte de la viscosité. & Effet de la houle sur le fond on sur les obstacles. & Très récent. \\
\hline Solution linéaire périodique. & Presque tous les problèmes pratiques. & Le plus ancien. \\
\hline Solution exacte périodique. & Quelques problèmes particuliers. & $\begin{array}{l}\text { Nouveaux développe- } \\
\text { ments récents. }\end{array}$ \\
\hline Solution irrégulière. & $\begin{array}{l}\text { Destinée vraisemblablement à se substituer à la } \\
\text { solution linéaire périodique dans la plupart } \\
\text { des problèmes. }\end{array}$ & Très récent. \\
\hline
\end{tabular}

\section{I. - EFFET DU VENT SUR LA MER}

Ce n'est qu'assez récemment qu'on a cherché à approfondir le mécanisme de formation de la houle et, du point de vue théorique, on ne peut pas dire qu'on ait beaucoup progressé.

Les résultats les plus tangibles de ces études sont les règles de prévision de la houle développées au cours de la dernière guerre mondiale.

Sous leur forme la plus élaborée, ces règles donnent, si l'on connaît à chaque instant la vilesse du vent en tout point de la zone de formation de la houle, non seulement l'amplitude et la période moyenne, mais aussi le spectre d'énergie, au sens qui sera exposé à propos de la représentation de houles naturelles.

Il serait imprudent d'ailleur's de limiter à l'établissentent de règles de prévision l'intérêt des études de l'effet du vent sur la surface de la mer.

Il est vraisemblable que certains effets des houles réelles sur le fond ou sur les ouvrages ne peuvent pas s'expliquer, si l'on fait abstraction du vent. Citons aussi que la reproduction de la houle sur modèle en faisant agir des ventilateurs, est peut-être la meilleure méthode pour obtenir des houles irrégulières analogues aux houles naturelles.

\section{II. - ETUDE DES HOULES FORMÊES}

\section{2-1. - Fluide réel}

\section{(prise en compte de la viscosité).}

La quasi-tolalité des études ne s'intéresse donc qu'à une houle déjà formée sans s'occuper de son origine et en supposant que l'air qui surmonte la surface est immobile et a une pression uni- forme. Une deuxième simplification aussi génćrale consiste à négliger la viscosité, c'est-à-dire à considérer l'eau comme un fluide parfait. Le très faible amortissement constaté dans la propagation des houles en nature et la belle régularité des trajectoires observées dans les canaux à houle étaient en faveur de cette hypothèse.

On se doutait pourtant qu'en faible profon- 
deur, elle n'était pas admissible au voisinage du fond.

Ce n'est qu'assez récemment que des études théoriques et des essais ont montré le rôle de la couche limite dans le déplacement des matériaux sur le fond par l'effet de la houle.

Un autre type de problème pour lequel la viscosité ne peut être négligée est l'étude des efforts subis par un corps placé dans la houle. Si l'on évalue cet effort en supposant le fluide parfait, on fait une erreur d'autant plus grande que les dimensions transversales du corps sont plus faibles. Ceci a été mis très clairement en évidence dans les études récentes relatives à la poussée subie par un cylindre vertical. Cette poussée s'évalue comme la somme de deux termes, l'un, proportionnel à l'accélération instanlanée de l'eau et au volume du cylindre, apparait dans l'étude théorique du problème par application des équations de l'hydrodynamique, l'autre au contraire, proportionnel au carré de la vitesse instantanée et à la section longitudinale du cylindre, est analogue à la traînée d'un corps placé dans un écoulement permanent.

\section{2.-2. - Fluide parfait.}

Négligeant l'effet du vent et de la viscosité, le mouvement doit satisfaire aux équations les plus simples de l'hydrodynamique.

On y ajoute la condition que le mouvement doit être irrotationnel.

Cette hypothèse est fondée sur le théorème de Lagrange sur la conservation de la nullité du rotationnel dans un mouvement où les forces de masse dépendent d'un potentiel.

La mer primitivement au repos ne peut prendre sous l'effet du vent qu'un mouvement irrotationnel.

Plus exactement, il en serait ainsi si les forces de viscosité étaient vraiment inexistantes. Bien que nous les négligions dans les équations, il sera tout à fail conforme aux raisonnements habituels de l'Hydraulique d'étudier des mouvements qui n'auraient pu apparaître en l'absence de ces forces de viscosité.

Cependant, l'hypothèse du mouvement irrotationnel demeure la base de départ des études. Rappelons, d'ailleurs, que la conservation du rotationnel étanl liée à la matière, la houle en se propageant peut cesser ou devenir rotationnelle suivant l'importance locale des forces de viscosité qui peuvent dépendre de l'action du vent ou de la profondeur.

Le mouvement irrotationnel d'un fluide parfait incompressible se traite le plus aisément dans le système des coordonnées d'Euler.

Le potentiel des vitesses est une fonction harmonique qui doit satisfaire aux conditions aux limites. Parmi celles-ci, la condition de surface, n'étant pas linéaire, introduit une grave complication. Non seulement on ne sait pas trouver de fonctions harmoniques $y$ satisfaisant, mais on ne pourrait pas les combiner pour obtenir la solution de problèmes particuliers.

\section{2-2-1. - SOLUTIONS LINÉaIRES périodiques.}

Pour supprimer cette difficulté, on remplace la condition de surface par une condition linéaire (la condition de Poisson) en négligeant les carrés des vitesses dans l'expression de la pression et en écrivant la constance de la pression non sur la surface libre, mais au niveau de repos; il faut reconnaître que cette simplification est assez lourde et les justifications qu'on en donne sont généralement très sommaires. En fait, sa meilleure justification est son intérêt pratique qui est considérable. On peut trouver, en effet, des solutions de ces équations simplifiées pour presque tous les problèmes et ces solutions donnent le plus souvent une très bonne approximation. Nous les appellerons solutions des équations linéarisées ou plus simplement solutions linéaires.

Nous mentionnerons d'abord la plus simple de ces solutions, la houle cylindrique fonction périodique du temps et de l'espace, sous ses deux aspects : houle progressive et houle stationnaire, chacune pouvant être considérée comme la combinaison de deux houles de l'autre sorte.

Toute une série de problèmes à trois dimensions relatifs au comportement de la houle en présence d'ouvrages maritimes, de la côte, de corps flottants, d'obstacles isolés quelconques, se traitent d'autre part en cherchant des fonctions harmoniques satisfaisant, outre à la condition de Poisson, aux conditions aux limites ci-dessous :

- Vitesse transversale nulle contre les obstacles;

- Mouvement se réduisanl à une houle cylindrique périodique à l'infini.

Des solutions exactes des équations linéarisées sont ou peuvent être trouvées (lorsque l'intérêt du sujet le mérite) à la plupart d'entre eux par l'emploi des méthodes générales de recherche des fonctions harmoniques qui constituent des outils très puissants.

Les combinaisons de telles solutions permettraient d'obtenir des solutions satifaisant à des conditions à l'infini aussi compliquées qu'on le voudrait.

Les développements mathématiques que ces recherches entraînent ne sont pas négligeables et la complexité des solutions esl souvent hors de proportion avec la précision qu'il est raisonnable de leur demander, compte tenu notamment de la nature des houles réelles, d'où l'inté- 
rêt des solutions approchées telle que la transposition de la méthode de Huyghens à l'étude de la réfraction, ou l'étude de la propagation de la houle à la côte par des considérations énergétiques.

\section{2-2-2. - Equations exactes.}

Comme nous l'avons dit, la justification de la linéarisation de la condition de surface ne peut se faire a priori et il est important d'indiquer quels problèmes font apparaître l'insuffisance des solutions qu'elle procure.

Parlons d'abord des conditions de déferlement. Il est évident qu'une approximation qui n'est certainement valable que pour des petits mouvements, est mal adaptée à renseigner sur les amplitudes maxima et, en fait, la houle linéaire que nous venons de définir ne présente pas d'amplitude limite. Toutefois, il existe une autre solution linéaire exprimée, celle-ci en coordonnées de Lagrange, qui présente au contraire une amplitude limite (d'ailleurs inexacte).

Ces deux solutions ont en commun que tous les éléments du mouvement (vitesse, dimensions des orbites, fluctuations de pression, etc.) sont proportionnels à une seule grandeur caractérisant l'amplitude du mouvement et sont des fonclions périodiques du temps. La première est la solution exacte des équations linéarisées; nous lui conserverons le nom de houle linéaire. La seconde est le premier terme dans la recherche d'une solution approchée des équations exactes et est appelée pour cette raison houle en première approximation. La première méthode est d'une application très générale, comme nous l'avons dit. La deuxième peut fournir une information plus riche sur un petit nombre de problèmes.

Lorsqu'on connail les deux solutions, elles ne diffèrent que par des termes dı deuxième ordre par rapport à l'amplitude du mouvement.

Le fait intéressant à signaler est que la houle en première approximation exprimée en coordonnées de Lagrange constitue une approximation nettement meilleure; outre l'existence d'une amplitude limite déjà citée, la surface libre est une trochoïde elliptique présentant la dissymétrie des crêles el des creux constatée en houle exacte alors que la surface libre de la solution en coordonnées d'Euler est une sinusoïde.

Utilisant cette remarque, $M$. Biesel a pu obtenir une représentation intéressante des conditions de déferlement sur une plage inclinée en approximation linéaire; mais il faut admettre que le fondement de cette méthode est difficile à justifier a priori.

Les études faites en dehors de l'approximation linéaire se développent dans deux directions :
- Existence et propriétés générales des ondes progressives périodiques d'amplitude finie satisfaisant exactement aux équations de l'Hydrodynamique;

- Recherche de solutions mieux approchées que l'approximation linéaire et appréciation de la validité de cette solution.

Bien entendu, cette division est très artificielle ou, plutôt, elle avait un sens lorsque les études sur les solutions exactes ne paraissaient pas susceptibles d'application pratique et que, d'autre part, on se préoccupait peu de la rigueur dans la recherche des solutions approchées.

Actuellement, la situation est différente et l'on peut dire que les deux sorles de recherches s'aident mutuellement.

Sur le premier point, on rappelle que l'existence d'ondes périodiques progressives cylindriques d'amplitude finie en profondeur constante a été démiontrée et qu'il est possible de calculer les caractéristiques de ces ondes sous forme de séries de puissances (1).

On a démontré également que le mouvement étant irrotationnel, les orbites ne pouvaient pas être fermées à tous les niveaux.

On s'est demandé si l'hypothèse du mouvement irrotationnel devait être conservée et si l'on ne devait pas admettre l'existence d'un rotationnel petit par rapport aux composantes de la vitesse.

Il existe donc une infinité de houles « exactes » correspondant à la même houle en première approximation.

Pour utiliser pratiquement ces résultats théoriques, on cherche des approximations meilleures que la première approximation, en supposant les formules développées en séries de puissance en $h / L$ et en calculant les lermes de proche en proche.

Parmi les résultats ayant déjà montré l'intérêt pratique de ces recherches, il faut rappeler qu'ayant établi une approximation au deuxième ordre de la houle, M. Miche a montré notamment qu'un terme du deuxième ordre, à période moitié de la période principale, pouvait avoir une grande importance dans l'évaluation de la pression au pied d'un mur réfléchissant la houle.

Cette prévision a été entièrenent vérifiée par les essais sur modèle qui ont été exposés dans une communication à la S.H.F.

Nous devons toutefois reconnaître qu'à notre connaissance aucune vérification en nature n'a été faite jusqu'ici, mais bien entendu il n'y a aucune raison qu'une telle vérification ne soit pas favorable.

(1) La seule autre sorte d'onde progressive d'amplitude finic dont on ait démontré l'existence est l'onde solitaire. 
Nous n'avons jusqu'ici parlé des houles non linéaires qu'à propos des phénomènes cylindriques et périodiques. Nous citerons pour terminer les recherches poursuivies actuellement $\dot{a}$ Grenoble pour composer ces houles de manière à obtenir des houles plus conformes aux phénomènes naturels. Cetle composition à la deuxième et même troisième approximation fournit des informations intéressantes qui ont fait l'objet de communications à la S.H.F.

\section{2-2-3. - Houles RÉELLES IRRÉguliÈres.}

Dans ce qui précède, nous avons exposé des résultats théoriques sans nous référer le moins du monde à des phénomènes naturels car, comme la remarque en a souvent été faite, les études théoriques sur la houle ont toujours été en avance sur les observations.

Supposons alors que nous soyons placés devant le problème pratique suivant : nous constatons une tempête sur une côte sauvage; quel sera l'effet de cette tempête sur les ouvrages que nous voulons réaliser pour y créer un port? Nous aurons ensuite à classer les tempêtes observées pendant une assez longue période, de manière à en faire une statistique et pouvoir en déduire des probabilités, mais il est évident que le premier problème à résoudre est celui de l'effet d'une tempête déterminée pendant son paroxysme. Tout $d$ abord, de quelles observations disposons-nous Mis à part le simple examen visuel de l'aspect de la mer, il n'existe guère qu'un moyen pratique et précis d'observation : c'est la mesure des fluctuations du niveau en un point; on peut le faire de nombreuses manières différentes (2), mais ceci ne change pas le fond du probleme el nous allons voir que cette information est bien maigre.

On a essayé, il esl vrai, d'oblenir une connaissance plus complete, en photographiant la surface de la ner de deux points élevés en restituant la surface suivant la mćthode ulilisée en lopographie. Mais ce procédé présente de très grandes difficultés pratiques et ne donne pas une grande précision. D'ailleurs, pour pouvoir reconstituer le mouvement de la mer, il faudrait obtenir une véritable cinéphotographic, ce qui n'a jamais été fait.

Ces photographies de la surface de la mer, les enregistrements des fluctuations de niveau, comme d'ailleurs la simple observation visuelle, suffisent cependant à nous montrer qu'en dehors de cas très particuliers, la surface de la mer ne ressemble pas du tout aux ondes périodiques

(2) I] y aurait beaucoup à dire, notamment sur la possibilité de reconstituer le mouvement de la surface à partir de Ia mesure des fluctuations de pression sur le fond. cylindriques et progressives qui ont été ctudièes en approximation linéaire, comme avec les ćqualions exactes.

Les phénomènes sont même si différents qu'on éprouve une certaine admiration pour la hardiesse de ceux qui ont établi un rapport entre eux.

Sur l'enregistrement des flucluations de niveau, on peut cependant distinguer des groupes d'ondulation relativement régulières et en déduire une amplilude et une période, puis assimiler le phénomène naturel à une onde progressive régulière ayant celte période el celle amplitude.

Cette méthode peul faire illusion quand on n'examine que l'enregistrement des fluctuations de niveau relativement régulier (surtout si e'est un enregistrement de pression sur le fond!) mais pas quand on peut disposer d'une pholographie instantanée de la surface.

On peut cependant la perlectionner en délerminant par un procédé moins arbilraire les caractéristiques de l'enregistrement.

On détermine, par exemple, les lois de distribution :

- de la dénivellation;

- de l'amplitude (comptée entre un creux el une crête conséculifs);

- des demi-périodes (mesurées entre une crête et un creux ou entre deux zéros); etc...

Sur un enregistrement assez long, cette étude fournit des résultats intéressanls. On constate par exemple que la loi de distribution des dénivellations est voisine d'une loi de Gauss et celle des amplitudes, voisine d'une loi de Rayleigh lice d'ailleurs à la première par une relation simple.

II semble donc que, dụ point de vue des amplitudes, un enregistrement peul se caractériser par un seul nombre.

Pour les périodes les résultals sont moins simples, mais on peut cependant espérer caractériser un enregistrement de houle par un petit nombre de grandeurs statistiques.

Mais ces considéralions ne nous autorisent nullement à dire que, pour un effet sur les ouvrages, la houle naturelle constalce est équivalente à telle ou telle houle périodi(fue progressive, par exemple celle ayant pour période la période moyenne de l'enregistrement et pour amplitude son amplitude significative, et pour cerlains problèmes on a même la preuve que celte équiralence n'est pas du lout juslifiée.

Au contraire, si nous pouvions montrer que la houle naturelle peut elre reproduite en composant des houles simples dont nous savons déterminer le comportement devant des ouvrages, nous disposerons d'une méthode de travail effi- 


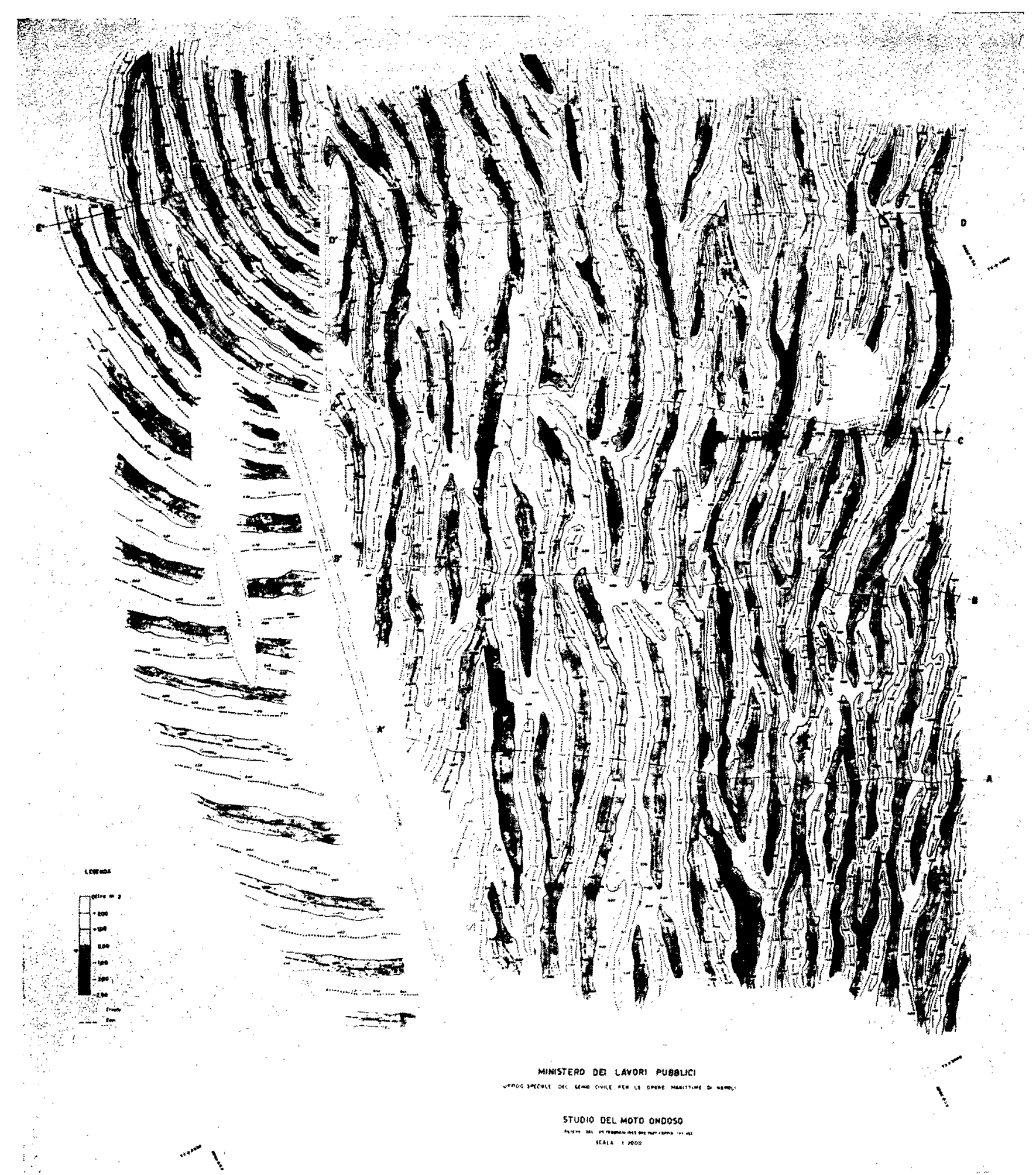

Restitution de la surtace de la mer par stérophotographic oblenue a la slation de mesure des efforts de la houle de Naples.

cace, mais dont nous voyons en même temps les limitations, car la composition de mouvements n'est justifiée que si les lois dont ils dćpendent sont linéaires. Cette condition a déjà été mentionnée. Elle est satisfaite dans des limites assez larges en profondeur constante, mais elle cesse certainement de l'être au voisinage d'une plage, d'un talus, d'une digue, etc...

Nous raisommerons d'abord sur une houle naturelle peu dispersée en direction, c'est-à-dire à longue crête; sans entrer dans les détails, il est évident en effet que la seule connaissance de la 
loi de dénivellation en un point, en fonction du temps, sera bien insuffisante pour nous renseigner avec précision sur une houle à trois dimensions et il est raisonnable, pour commencer, de limiter notre ambition.

Ayant donc observé une loi de hauteur $/(t)$ pour $t$ compris entre $O$ et $D$, peut-on la considérer comme produite par la composition d'un nombre fini ou infini de houles élémentaires, la surface libre de chacune d'elles étant donnée par :

$$
\eta(x t)=\mathrm{A}(\mu) \cos \left[\left(\mu^{2} / g\right)\right] x-\mu t+\varphi(\mu)
$$

Si cela est possible, on doit pouvoir déterminer $A(\mu)$ et $\rho(\mu)$ pour que :

$$
f(t)=\Sigma \mathrm{A}(\mu) \cos [\mu t-\varphi(\mu)]
$$

pour $t$ compris entre $O$ et $\mathrm{D}$.

On cherche d'abord à réaliser cette identité en sommant des houles composantes dont les périodes sont des sous-multiples de D.

Cette méthode n'est pas retenue en pratique pour différentes raisons dont la principale est au fond qu'il faut, pour reproduire $f(t)$ avec une assez bonne approximation, un grand nombre de composantes dont les amplitudes et les phases, ne pouvant être définies par des lois simples, constitueraient une masse de données impossibles à manier.

On pourrait prévoir qu'il en serait ainsi car, comme nous l'avons dit, la loi de distribution des dénivellations d'une houle réelle est Gaussienne alors que, pour une sinusoïde, c'est une fonction à deux points infinis et qu'il faut combiner un très grand nombre de fonctions sinusoïdales pour obtenir une loi de distribution se rapprochant de la formule de Gauss.

Une deuxième méthode consiste à représenter $f(t)$ par une intégrale de Fourier où $\mathrm{A}(\mu)$ et o (u) seraient des fonctions de $\mu$. de forme simple dépendant d'un petit nombre de paramètres.

On notera, toul d'abord, que la seule connaissance de $f(t)$, même pendant un temps infini, ne suffirait à déterminer exactement A ( $\left.\mu_{0}\right)$ et $\rho_{(}\left(\mu_{0}\right)$ qu'à condition de supposer que loutes les ondes se propagent dans le même sens, mais même en faisant cette hypothèse, $f(t)$ n'étant connu que pendant un temps assez bref, on ne pourra jamais connaître exactement $A$ el $\varphi$.

Il ne faut pas trop le regretter, ni s'en étonner, si l'on pense que la connaissance exacte de $A$ et $\varphi$ permettrait de connaitre la dénivellation en tout temps et en tout lieu, ce qui est à la fois trop ambilieux et inutile, car ce qui nous intéresse ce n'est pas de reconstituer exactement

(3) Cette formule est valable en profondeur infinie. En profondeur finie, le coefficient de $x$ serait une fonclion de $\mu$ qu'on ne sait pas expliciter.
《I'histoire » d'une tempête, c'est-à-dire la loi exacte de la dénivellation en fonction du temps, mais des propriétés à caractère général.

Si l'on essaie, non pas de reconstituer théoriquement les fonctions $A$ et $o$, mais de choisir a priori des fonctions simples dépendant d'un petit nombre de paramètres et de déterminer ces paramètres en essayant d'ajuster au mieux l'intégrale de Fourier à la loi expérimentale $f(t)$, on n'obtient pas une loi assez irrégulic̀re pour représenter $f(t)$.

Il existe enfin un troisième point de vue : nous pouvons supposer que les valeurs de $A$ el $\varphi$ sont, pour chaque valeur de p., déterminées par un tirage au sort.

La houle sera alors caractérisée, non par des fonctions dont nous avons montré qu'elles ne pouvaient pas être simples, mais par des lois de probabilités.

C.e schéma sera intéressant si on peut obtenir une bonne représentation de la houle naturelle avec des lois de probabilité simples et s'il donne lieu à des développements théoriques conduisant à des applications pratiques.

Or, il se trouve qu'effectivement on peut représenter la houle réelle par des lois de probabilité très simples et que celte représentation se raltache à la théorie des fonctions aléatoires qui s'est développée notamment autour de la théorie du bruit en électronique.

L'étude, même sommaire, de la représentation de la houle naturelle par schéma aléatoire nous entraînerait beaucoup trop loin. Nous n'en rappellerons que quelques aspects élémentaires.

\section{a) JUSTIFICATION THÉORIQUE DU SGHÉMA ALÉATOIRE DE REPRÉSENTATION DE LA HOULE (analogie avec la théorie du bruit).}

On considere que l'action sur la mer peut se représenter par une suite d'impulsions identiques, de sorte que l'agitation en un point assez éloigné de la zone de génération peut être considérée comme la superposilion de trains de houles identiques, chacun d'eux résultant d'une impulsion déterminée.

L'instant exact d'apparition de chaque impulsion et, par conséquent, de chaque train, est un événement qu'il est inutile de connaître, car il est propre au cas particulier de chaque tempête; par contre, si l'on suppose que ces impulsions sont distribuées dans le temps indépendamment les unes des autres, avec une densiti de probabilité uniforme dépendant de l'intensité du vent, ef, si l'on connaît les caractéristiques des trains de houle élémentaires, dépendant aussi de l'intensité du vent, on concoit $q u$ 'on puisse déterminer certaines caractéristiques communes 
à toutes les tempêtes qui peuvent être engendrées par un vent de vitesse moyenne déterminée.

Tous les enregistrements obtenus en un point pour toutes les tempêtes engendrées en des zones voisines par des vents identiques pourront être considérés comme des réalisations différentes d'une même fonction aléatoire.

Puisqu'on considère seulement la partie de la tempête où la densité de probabilité des impulsions ne varie pas, la représentation de l'agitation possède les propriétés suivantes :

- Les statistiques sont indépendantes du temps (caractère stationnaire);

- Les statistiques faites sur différentes réalisations possibles sont équivalentes aux statisti- ques faites au cours du temps (propriété ergodique) - propriété très générale des fonctions aléatoires stationnaires qui nous permettra de confondre les valeurs moyennes par rapport au temps et les espérances mathématiques relatives à différentes réalisations possibles du phénomène.

Si $\mathbf{R}(t)$ est l'enregistrement qui résulterait d'une seule impulsion à l'instant $O$, l'enregistrement relatif à l'agitation totale peut être représenté par :

$$
\eta(t)=\Sigma \mathrm{R}\left(t-t_{j}\right)
$$

où les $t_{i}$ sont les instants successifs d'apparition des impulsions.

On peut décomposer $\mathrm{R}(t)$ fonction certaine et supposée connue, en série de Fourier, soit :

$$
\begin{gathered}
\mathrm{R}(t)=\Sigma \mathrm{A}_{p} \cos \left(p k t-\varphi_{p}^{\prime}\right) \\
\eta(t)=\sum_{p=1}^{p=\infty} \mathrm{I}_{p} \cos \left(p k t-\varphi_{p}\right) \text { arec } \mathrm{I}_{p} \cos \left(p k t-\varphi_{p}\right)=\sum_{j=1}^{j=\mathrm{N}} \mathrm{A}_{p} \cos \left(p k t-p k t_{j}+\vartheta_{p}^{\prime}\right)
\end{gathered}
$$

$\mathrm{I}_{p}$ et $\varphi_{p}$ sont l'amplitude et la phase de la résultante de $\mathrm{N}$ vecteurs égaux dont les phases sont distribuées uniformément entre 0 et $2 \pi$.

On montre que l'amplitude $I_{p}$ est une variable aléatoire distribuée suivant la loi de Rayleigh et que la phase $\varphi_{p}$ est distribuée uniformément entre 0 et $2 \pi$.

Ia valeur moyenne du carré de $I_{p}$ est $\Sigma \mathbf{A}_{p}{ }^{2}$, et cette constatation est un aspect de la proposition suivante, qui explique toute la formulation intervenant dans la représentation aléatoire de la houle.

«En probabilité, ce sont les carrés des amplitudes (donc les « énergies ») qui s'ajoutent et non les amplitudes. 》

\section{b) Formulis :}

L'extension de la formule ci-dessus à une densité très grande d'impulsions conduit à écrire :

$$
\eta(t)=\int_{0}^{\infty} \cos \left(k l \cdot \ldots \psi_{k}\right) \mathrm{I}_{k} d k
$$

oun $\mathrm{I}_{k} d k$ et sont des variables aléatoires dont les lois de distribution sont :

pour $\mathrm{I}_{l i}$ dli :

$$
\frac{2 \mathrm{I}_{k} d k}{\mathrm{E}_{k} d k} e^{-\frac{\left(\mathrm{I}_{k} d k\right)^{2}}{\mathbb{E}_{k_{i}} d l_{k}}} d\left(\mathrm{1}_{k i} d k^{k}\right)
$$

pour $\psi_{k}:$ distribution uniforme entre 0 el $2 \pi$.

Mais il ne faut pas perdre de vue que l'expression ci-dessus n'est pas une formule calculable, aussi n'est-il pas indispensable de l'écrire.

La seule caractéristique dont dépend le phénomène est en définitive $\mathrm{E}(k) d k$, c'est-à-dire la valeur moyenne de $\left(\mathrm{I}_{k} d k\right)^{2}$ ou encore l'énergie moyenne des composantes dont le nombre d'onde est compris entre $k$ et $k+d k$, c'est-à-dire finalement la fonction certaine $\mathrm{E}(k)$ qu'on appelle souvent le spectre d'énergie.

On trouve cependant dans les ouvrages américains l'expression :

$$
\eta(t)=\int \mathrm{A}(k) \cos \left(k t+\varphi_{k}\right) \sqrt{d k}
$$

Dans cette expression (qui n’est pas plus calculable que la précédente), A ( $k$ ) est la fonction certaine $\sqrt{\mathrm{E}(k)}$. 
Cette expression est moins générale que celle donnée précédemment, puisque seule la phase des composantes est aléatoire, mais elle paraît avoir une généralité suffisante en pratique pour représenter les houles naturelles (4).

Il faut rappeler d'autre part qu'il n'y a entre la formule dite de l'intégrale de Fourier el l'expression indiquée ci-dessus, qu'une analogie formelle.

\section{INTÉgrale de Fourier}

$\eta(t)=\int \mathrm{E}(k) \cos \lfloor k t+\varphi(k)] d k$

$\mathrm{E}(k)$ et $\varphi(k)$ sont des fonctions.

Formule calculable.

$\eta(t)$ tend vers 0 lorsque $t \rightarrow \infty$.

$\int r_{1}^{2}(t) d t$ a un sens (énergie totale finie).

Observation. - Il n'est pas impossible a priori que les $\varphi_{k}$ représentent la suite de valeurs de $\varphi(k)$, mais la probabilité d'un tel événement est évidemment nulle.

Pour achever, nous rappellerons enfin la formule essentielle suivante :

La fonction spectrale (ou spectre d'énergie) est la transformée de Fourier de la fonction d'autocorrélation :

$$
\overline{\eta(t) \eta(t+\mathrm{T})}=\int \mathrm{E}(k) \cos 2 \pi k^{\prime} \mathrm{T} d k
$$

et notamment :

$$
\overline{\eta^{2}(t)}=\int \mathrm{E}(k) d k
$$

Ceci est une propriété générale des fonctions aléatoires stationnaires.

c) VÉRIFICATION DE LA VALIDITÉ DE LA HOULE PAR DES PROCESSUS ALÉATOIRES PAR COMPARAISON AVEC LES PROPRIÉTÉS STATISTIQUES DE HOULES RÉELLES.

Nous avons cité tout à l'heure quelques propriétés statistiques des enregistrements de houle et montré qu'elles étaient en contradiction avec

\section{REPRÉSENTATION ALÉATOIRE}

$\eta_{i}(t)=\int \mathrm{A}(k) \cos \left(k t+\varphi_{k}\right) \sqrt{d k}$

A $(k)$ est une fonction, mais $\varphi_{k}$ est une variable aléatoire prenant n'importe quelle valeur entre 0 et $2 \pi$.

Formule non calculable.

Phénomène stationnaire.

Energie totale infinie.

leur représentation par la somme d'un petit nombre de composantes.

Au contraire, ces propriétés s'accordent très bien avec la représentation de la houle par un schéma aléatoire, ce qui en est la meilleure justification.

d) Extransion a la houle a trois dimensions.

On peut généraliser le schéma ci-dessus en écrivant (Pierson) :

$$
\begin{aligned}
& \eta(x y t)=\int \mathcal{A}(k, 0) \\
& \cos \left[k^{2} / g(x \cos 0+y \sin 0)-k l+\varphi_{k, 0}\right] \sqrt{d k d 0}
\end{aligned}
$$

La houle est représentée par la somme d'ondes élémentaires de périodes et de directions différentes, l'énergie des ondes dont la période et la direction sont comprises entre 0 et $\theta+d \theta$, et $k$ : el $k+d k$ est $\mathrm{A}^{2}(k, 0) d k d \theta$.

On peut aussi, comme Longuet-Higgins, prendre pour fonction spectrale $\mathrm{E}(u b)$ avec (en profondeur infinie) $u=\left(k^{2} / g\right) \cos 0$ et $\mathrm{V}=\left(k^{2} / g\right) \sin 0$.

(4) On peut justifier l’écriture $\sqrt{a k}$ en définissant l'intégrale ci-dessus à la manière de l’intégrale de Riemamn par la limite d'une somme.

Si $A(k)$ est une fonction certaine et si $\varphi s$ prend n'importe quelle valeur comprise entre 0 et $2 \pi$ pour chaque valeur de $k$, une expression telle que :

$$
\Sigma A(k) \cos \left(k t+\varphi_{k}\right) \Delta k
$$

aurait pour n'importe quelle valeur de $t$ une probabilité nulle d'être différente de 0 .

En effet, si $\mathrm{A}(k)=\mathrm{C}^{\mathrm{te}}$, on peut écrire :

$$
\int A \cos \left(k t+\varphi_{k}\right) d k=\operatorname{limite}_{N \longrightarrow \infty}(A / N) \Sigma \cos \varphi
$$

Mais la déviation type de la somme des $\mathrm{N}$ variables aléatoires $\cos \varphi$ croît comme $\sqrt{\mathrm{N}}$, donc la déviation type de $\int \cos \varphi d k$ est nulle, alors que celle de $\int \cos \varphi \vee \sqrt{d k}$ est finie. 
Ces formules fournissent des données statistiques sur la variation du niveau en un point et sur la forme de la surface. Pour ces dernières, on ne peut malheureusement guère faire de vérificalion faute d'observations en nature. Tout ce qu'on peut dire c'est que les observations en nature n'ont pas contredit la représentation aléatoire de la houle, mais non qu'elles ont prouvé sa valeur. On ne sait d'ailleurs rien ou presque rien de la corrélation spatiale de l'agitation.

\section{e) DÉtermination DE la Fonction spectrat,e.}

En définitive, c'est elle qui caractérise la houle. Etant donné la définition aléatoire du phénomène el la brièveté de l'échantillon cu'on en possède, on ne pourra avoir qu'une connaissance approchée de cette fonction, mais on sera en mesure d'évaluer l'erreur possible sur sa détermination.

D'ailleurs, un seul enregistrement ne permet de déterminer que :

$$
E(k)=\int_{-\pi}^{+\pi} A^{2}(k \theta) d \theta,
$$

et non la véritable fonction spectrale $\mathrm{A}^{2}(k \theta)$; autrement dit, nous ne savons rien de la dispersion en direction de la houle, ou, ce qui revient au même, de la longueur des crêtes.

Certaines méthodes permettant de donner un ordre de grandeur de la dispersion en direction sont basées sur l'examen de la réflexion du soleil dans la mer.
On peut déterminer ainsi l' «allongement des crêtes $\gg 1 / \gamma$ défini par :

$$
\gamma^{2}=\frac{m_{02}}{m_{20}}
$$

où $m_{02}$ et $m_{20}$ sont les moments du 2 ordre de la distribution de $\mathrm{E}(u \mathrm{~V})$ (fonction spectrale dans la représentation de Longuet-Higgins).

\section{f) Problèmes pratigues.}

Peu de problèmes pratiques paraissent encore avoir été traités dans la représentation aléatoire de la houle.

Les plus faciles seront ceux ò̀ l'effet à étudier provoquera sur la houle une action linéaire, c'est-à-dire, au fond, tous les problèmes qui peuvent être résolus exactement dans l'approximation linéaire (réfraction et diffraction notamment).

L'effet sera alors assimilable à celui d'un filtre linéaire et la houle en un point sera caractérisée par la fonction spectrale $g^{2} k \mathrm{E}(k)$ où $g(k)$ est le gain du filtre.

\section{g) INSUFFisANCE DE LA REPRÉSENTATION ALF́a-} TOIRE DES HOULES.

Les deux principales insuffisances paraissent les suivantes:

- C'est une théorie linéraire inapplicable dès que lamplitude devient notable et que la profondeur devient trop faible.

- On manque totalement d'informations en nature pour la justifier et l'appliquer aux phénomènes $\dot{a}$ trois dimensions.

\title{
C O M M E N T A I R E
}

\author{
par M. Chapouthien
}

Président de la séance

M. le Président remercie M. Banal d'avoir réussi à mettre de l'ordre et de la clarté dans des problèmes complexes et difficiles.

«J'ai été, dit-il, très sensible à l'élégance de l'itinćraire infellectuel suivi par les spécialistes de la houle, tel que M. Banal vient de nous le décrire. Il correspond à un schéma assez général que l'on retrouve aujourd'hui dans plusieurs chapitres de la Physique.

«Certains aiment à répéter que l'esprit humain, comme l'humanité, passe successivement par trois états: l'àge de la règle de trois, l'àge de la fonction, l'âge de la probabilité.

«L'âge de la règle de trois correspond à la fonction linéaire; on linéarise la condition de Poisson et e'est sur des approximations linéaires que l'on commence à se faire la main.

« Mais ces approximations ne suffisent pas : vient alors l'áge de la fonction; on tente de résoudre l'équation de Laplace, de trouver des schémas analyticues rigides et rigoureux; on obtient tout le bel ensemble d'études qui va de Von Gerstner à Stokes.

"Comme la solution fonctionnelle usuelle est cllemême imparfaite, on a recours à des développements en série qui, progressivement, nous acheminent vers le troisièmc schéma, celui atuquel nous n'acédons qu'assez tard dans notre maturité : le schéma aléatoire régi par un lien causal plus souple et plus nuancé, schéma qui parait donner les explications les plus conformes aux observations pratiques.

«La nature est trop riche pour rester linéaire; elle n'est pas non plus aussi rigide que nous l'avons cru tout d'abord, mais elle accepte de se conformer assez bien au schëma aléatoire : moyens écarts, valeurs probables, corrélations envahissent maintenant la physique et l'art même de l'Ingénieur. » 